\title{
Quality of Life after Cardiac Rehabilitation for Patients with Heart Failure: 18 months follow-up
}

\author{
Elias F. Porto ${ }^{1}$, Claudia Kümpel ${ }^{1}$, José R. Leite ${ }^{2}$, Aline A. Andrade ${ }^{1}$,Natália C. Oliveira ${ }^{3,4}$, \\ Leslie A. Portes ${ }^{1,3,4, *}$ \\ ${ }^{1}$ Physical Therapy Department, Universitary Clinic, Adventist Univesity of São Paulo (UNASP), Brazil \\ ${ }^{2}$ Clinicas Hospital from University of São Paulo Medical School, Brazil \\ ${ }^{3}$ Exercise Physiology Laboratory (LAFEX-UNASP), São Paulo, Brazil \\ ${ }^{4}$ Physical Education Department, Adventist University of São Paulo (UNASP), Brazil
}

\begin{abstract}
The aim of this study was to evaluate the effects of a supervised cardiac rehabilitation (CR) program on longterm quality of life (QoL) of patients with heart failure (HF).Methods: A sample of 21 HF patients, previously sedentary, NYHA II, was randomly divided into two groups: the CR group $(\mathrm{N}=13)$ and the control group $(\mathrm{CG}=8)$. The CR consisted of sessions of 120 minutes/day, 3 times/week,for 60 days. CR group performed their activities under supervision at a rehabilitation clinic, and CG received standard care and was instructed to exercise at home. All patients underwent a maximal symptom-limited test at the beginning and after 30 and 60 days of CR. QoL was monitored after 60-days, 6, 12 and 18 months of CR. Results:Two-way A NOVA for repeated measures, followed by Tukey's test for statistical analysis showed significantimp rovement in peak $\mathrm{VO}_{2}$ at 30 and 60-days of CR $(\mathrm{p}<0.05)$. QoL improved after 60-days of CR and remained improved throughout the 18following months in the supervised CR group. The use of hospital emergency services became less frequent $(\mathrm{p}=0.023)$ and there was a trend for better survival after 18 months of follow-up $(p=0.092)$.Conclusion: CR was effective upon cardiopulmonary and Qo L aspects.
\end{abstract}

Keywo rds Heart Failure, Cardiac Rehabilitation, Functional Capacity, Quality of life, Survival

\section{Introduction}

Heart Failure (HF) is, in most of the cases, a status due to the evolution of a number of illnesses, such as arterial hypertension or high blood pressure, coronary heart disease (CHD), acute myocardial infarction (AMI), valvediseases and cardiac arrhythmic illnesses[1,2]. The main HF symptoms are dyspnea, physical activity intolerance and quality of life reduction[2,3]. HF represents the main cause for hospital admission in individuals aging 65 and beyond. It is a fact that HF prevalence is increasing, and among the factors that might be contributing to this situation we highlightthe increase in life expectancy of the general population and greater effectiveness of new drugs[4]. However, despite the advent of new drugs, mortality and morbidity indexes are still high in this type of affection[1,2]. Cardiac rehabilitation (CR) has become mandatory in HF patients. Objectives of CR include mortality and morbidityreduction, quality of life improvement and cos reduction[5-13].

* Corresponding author:

leslie.portes@unasp.edu.br (Leslie A. Portes)

Published online at http://journal.sapub.org/ajmms

Copyright (C 2012 Scientific \& Academic Publishing. All Rights Reserved
Several studies have already demonstrated the efficacy of exercise - based CR programs [7-11], but a meta - analysis of randomized controlled trials[12] has concluded that quality of life improves to similar levels in patients receiving $\mathrm{CR}$ and standard care.The aim of the present study was to evaluate the effectiveness of asupervised CR program in the quality of life of HF subjects, as compared to standard care. Success indicators were based on cardiorespiratory capacity analysis, functional capacity, quality of life, survival and/or mortality.

\section{Methods}

The present study was based on a longitudinal prospective design composed by two phases: $1^{\text {st }}$-supervised CR or non-supervised CR (control) for 60 days; and $2^{\text {nd }}-18$ months follow-up after CR. All procedures in the study were in conformation with resolution 196/96 of the Brazilian Health Council(http://conselho saude.gov.br/resol ucoes/1996/Reso196.doc) and the declaration of Helsinki[14], and were approved by the local University ethics committee (protocol number: 004/2005).

\subsection{Patients}

After discharge from hospital, 28 male patients 
volunteered to participate in the study. They were rando mly distributed into two groups: CR $(n=16)$ and $C G(n=12)$. Random distribution was performed as follows: for each participant that enrolled the study, researchers drew out a number from a box (1 or 2 ), and this consequently led to different group sizes.

\subsection{Selection Criteria}

At the beginning of the study, we excluded from our sample subjects in whom the following incidents had occurred less than three months ago: myocardial in farction, ischemic myocardial manifestation,unstable angina anduncontrolled high blood pressure. We also excluded subjects who were physicallyactive before the cardiovascular event, the ones who were physically impaired due to brain vascular disease,orthopedic or rheumatologic limitations, and individuals with lack of availability to participate. After being adequately informed about all the procedures in the study, all subjects signed aninformed written consent. Twenty-one out of 28 patients concluded the CR program, 3 patients were excluded from $\mathrm{CR}$ and 4 from the CG. Patients were excluded because of cardiac arrhythmia $(n=2)$, because they did not take personal medication adequately $(\mathrm{n}=2)$ one of themreferring sporadicangina pectoris, and 3 were excluded because of lack of availability to participate in the study. Final group numbers were 13 subjects in CR and 8 in CG. All 21 patients had New York Heart Association functional classification II (NYHA II)[15]. These individuals had clinic and hemodynamic stability according to the "American Heart Association"[13,15]and had not been hospitalized 6 months previously to the beginning of the study. All of them were instructed not to suspend medication intake during the study period, except by medical prescription.

\subsection{Cardiopulmonary Evaluation}

Patients underwent cardiopulmonary tests in 3 distinguished moments: one week before initiating participation, 30 days and 60 days after the beginning of the study. Metabolic measures were obtained by means of K4b2 Cosmed ${ }^{\circledR}$ gas analyzer, model CO9052-02-99 (Rome, Italy).Throughout the tests,electrocardiographic trace (ECG) and peripheral oxygen saturation $\left(\mathrm{SpO}_{2}\right)$ were constantly monitored; and arterial blood pressure was measuredevery 3 minutes. Dyspnea sensation was also verified every minute using Borg scale[16,17]and ventilation and metabolic variables were acquired breath by breath. All tests were carried out on a treadmill protocol according to maximum test symptom limited[17]. Treadmill speed was chosen for each patient and was maintained unaltered until the end of the test. During the first three minutes of the test the treadmill inclination was zero and from the fourth minute onwards the treadmill inclination increased by $1 \%$ every minute until the end of the test $[16,17]$. The duration of incremental test should be between 8 and 12 minutes. When the test lasted less than 8 or more than 12 minutes, a new test was performed the next day. The speed of the treadmill was based on the evaluator's experience.

\subsection{Training Protocol}

CR and CG patients were accompanied by a professional team during the study period. CR performed approximately 20 minutes of upper and lower limbs calisthenic exercises. After that, patients walked for 30 minutes on the treadmill, with inclination being adjusted at moment zero and maintained until the end ofthe training session. Regarding speed, the first three and last five minutes were set at 1.6 $\mathrm{km} / \mathrm{h}$ for warm up and cool down, respectively. The intermediate 22 minutes were effectively dedicated to training with customized load. Heart rate was continuously monitored during training by a Polar ${ }^{\circledR}$ heart rate wrist monitor. $\mathrm{SpO}_{2}$, breath rate and dyspnea sensation were also observed. In case of $\mathrm{SpO}_{2} \leq 88 \%$, enough oxygen through nasal catheter or Venturi mask was given to the patient to maintain $\mathrm{SpO}_{2} \geq 92 \%$. CR program period was 60 days, 3 times a week, 120 minutes/session, divided into10 minutes of stretching exercises, 20 minutes of calisthenic exercises, 3 minutes of warm-up in treadmill, 22 minutes of treadmill exercise as described above, 5 minutes of cool down, 20 minutes of stretchingexercises and the last 30 minutes were dedicated to rest before being dismissed to go home. Treadmill training load was individualized,based on initial cardiopulmonary test. Exerc ise intensity was set to maintain HR between $60 \%$ and $70 \%$ of the maximum obtained in the test.

Throughout the training, due to the individual's physical capacity improvement, perceived by decrease indyspnea and a better well-being sensation, thepercentage of the training load was increased up to $80 \%$ maximu m HR limit.

Patients of CG received standard care: orientation regarding benefits promoted by physical activities and the necessity and importance that all individuals performed the proposed activities regularly. Subjects received instructions to exercise3 times/week, 120 minutes/sessionduring 60 days. Patients should walk for30 minutes in a rhythm that was enough to keep their HR between $60 \%$ and $70 \%$ of maximum. Besides that, patients received instructions on how to perform warm upand back to rest activities. To do so, all patients received a $\operatorname{Polar}^{\circledR}$ heart rate monitor and received instructions regarding HR intensity and monitoring. Every CG patient was informed about how to complete calisthenic exercises. All patients were required to complete a diary log with the number of daily training hours, and to take notes of possible unexpected events during the walking sessions.

Once a week, patients of both groups attended educational lessons about themes such as tobacco, alcoholism, high blood pressure, diabetes mellitus and proper use of medicine. Both groups were giventhe schedules in advance for all of the lectures.One day before 
each class all patients received phone calls reminding them of the rehabilitation center visit.

\subsection{Quality of Life and Symptoms Evaluation}

Symptoms and quality of life were evaluated by using the Minnesota Living with Heart Failure Questionnaire (MLwHFQ)[18]applied at the beginning, after 60 days, 6 months, 12 months and 18 months of follow-up. The MLwHFQcontains 21 questions, each one with 6 alternatives to choose from: no, very little, little, more or less, much and very much; with "no" corresponding to zero and "very much" matching5. Finally, the nu mber of hospital admissions, emergency room attendances and mortality were evaluated monthly.

\section{Statistical Analysis}

Results are described as mean \pm standard deviation. Initially, groups were compared by the non-parametric Mann-Whitney test. The excluded patients' data was compared to the ones of those who completed the study by the Wilcoxon test. Two-way ANOVA for repeated measures was adopted, followed by Tukey's test for comparisons between CR and CG, considering the following factors: exerc ise train ing program (CR versus $\mathrm{CG}$ ) and time (beginning, 30 days, 60 days, 6, 12 and 18 months follow-up). Mortality was evaluated according to the Kaplan-Meier method[5]. Analyses were made by Sig maStat3.5 (www.Systat.com). In all cases, statistically different results were taken into account if $\mathrm{p}<0.05$.

\section{Results}

Data fro $m$ the 21 patients who comp leted the intervention protocol did not differ statistically from the 7 patients who were excluded from the study (data not presented). TABLE 1 shows both study groups results before CR. No statistically significant differences were found between CR andCG regarding age, maximum testtime length, peak heart rate (peak HR), peak $\mathrm{VO}_{2}$ and most frequent cardiac insufficiency aetiologies (high arterial blood pressure, valvedisease and acute myocardial infarction). Ninety-two percent of CR patients performed all sessions proposed. Although all patients in the control group received phone calls and encouragement to accomplish the physical activities proposed, only $16 \%$ performedthe activities as recommended, 35\% performed them partially and $49 \%$ did not perform the activities as recommended.

FIGURE 1A illustrates results relative to maximum amount of time on treadmill test for the two study groups. CR and CG patients were compared in itially at 30 and 60 days of their respective programs. Statistically significant influences were observed regarding the program (CR versus CG) and the interaction (program plus period of time) in relation to time length of maximum test. The influence of time (beginning versus 30 versus 60 days) was observed in CR group (beginning $<30$ days $<60$ days, $\mathrm{p}<0.001$ ) and
inCG (beginning $<30$ days $=60$ days, $\mathrm{p}<0.004$ ). FIGURE 1B illustrates results relative to peak heart rate (peak HR) for both study groups. Statistically significant differences regarding time were observed only in CR group (beginning $>30$ days $=60$ days, $\mathrm{p}<0.027$ ). Values obtained fromCG (beginning $=30$ days $=60$ days, $p>0.05$ ) were not significantly influenced by the amount of time required for the test to be performed, and in the same way there were no significant differences between CR and CG in each moment throughout the programs. FIGURE 1C illustrates peak $\mathrm{VO}_{2}$ results for both study groups. Statistically significant in fluences of time were observed in both groups: CR: beginning $<30$ days $<60$ days $(\mathrm{p}<0.001)$; CG: beginning $<30$ days $>60$ days $(\mathrm{p} \leq 0.008)$. When comparing groups it was noticed that at 60 days CR peak $\mathrm{VO}_{2}$ values were significantly higher than CG $(\mathrm{p}=0.027)$. Additionally, rehabilitation programs did not result in patients' functional classification change.

Table 1. Chronic heart failure patients' characteristics

\begin{tabular}{|c|c|c|c|}
\hline Variables & CR & CG & Significance \\
\hline Age (years) & $52.4 \pm 2.4$ & $53.1 \pm 1.8$ & NS \\
\hline \multicolumn{4}{|c|}{ Ergospirometry } \\
\hline $\begin{array}{c}\text { Test time extent } \\
\text { (minutes) }\end{array}$ & $9.80 \pm 0.94$ & $9.20 \pm 0.81$ & NS \\
\hline Peak HR(bpm) & $136.8 \pm 16.5$ & $138.3 \pm 12.0$ & NS \\
\hline $\begin{array}{c}\text { Peak VO } \\
\text { (ml/kg/min) }\end{array}$ & $13.9 \pm 3.7$ & $13.2 \pm 3.2$ & NS \\
\hline \multicolumn{4}{|c|}{ Etiology (\%) } \\
\hline HBP & 54 & 38 & NS \\
\hline Valvar & 15 & 12 & NS \\
\hline CAD & 31 & 50 & NS \\
\hline
\end{tabular}

Data expressed as mean \pm standard deviation. CR: supervised cardiac rehabilit ation or CG: control group program. HR: heart rate, HBP: high blood pressure, CAD: coronary artery disease. NS: statistically non-significant $(p>0,05)$.

The compared average hospital admission frequency of both groups during 60 days of CR did not differ statistically: CR (0.39 times) and CG (0.75 times), but the average CR emergency room attendance ( $0.6 \mathrm{v}$ isits) was significantly lower ( $\mathrm{p}=0.023)$ when co mpared to CG (1.6 visits).

There was no death incident at the end of the 60 days of CR training in both groups. After 18 months of follow-up, the number of deaths did not differ $(p=0.092)$ when comparing both study groups: CR: 1 death and CG: 4 deaths. Survivalwas $92 \%$ and $50 \%$ at the end of the 18 months period, respectively $(\mathrm{p}=0.092)$.

The results regarding symptoms presence evaluated by the MLwHF in both groups are illustrated in FIGURE 2. Two-way ANOVA identified program effect $(\mathrm{p}<0.001)$ and follow-up time effect $(\mathrm{p}<0.0001)$ in both groups. CR scores improved after 60days intervention and kept lower (better) than the previous CR scores until 18 months followup ( $<<0.0001)$. CGscores did not change from the beginning until 60 days of program and got significantly worse when comparedto initial numbers and the ones verified at 6, 12 and 18 months follow-up $(p=0.022)$. Better scores were observed at 60 days, 6, 12 and 18 months of intervention in CR group when intergroup comparisons were made (Tukey's test, $\mathrm{p}<0.001$ ). 

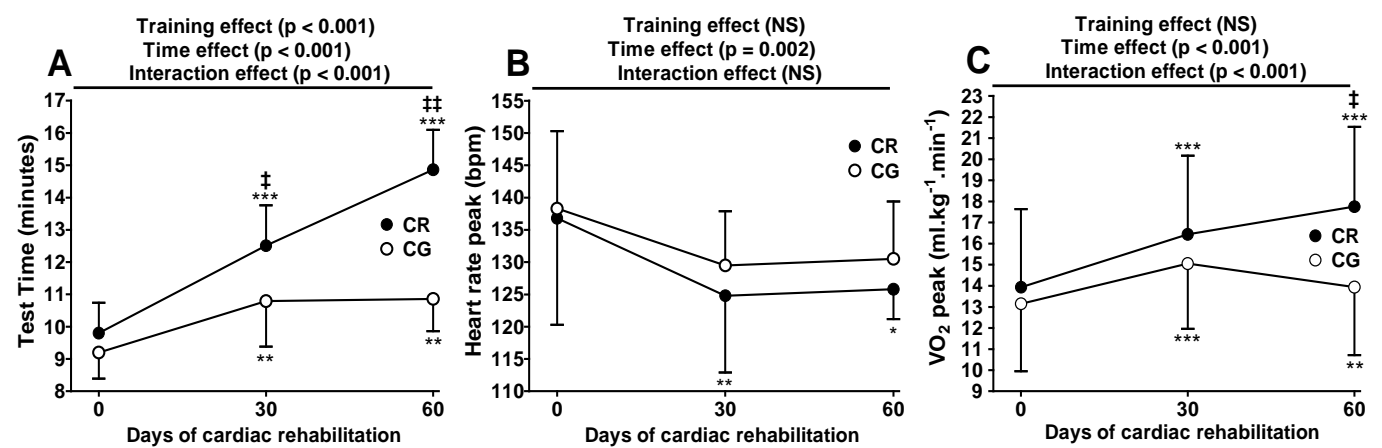

Figure 1. Supervised cardiac rehabilitation (closed symbols) and control (open symbols) effects in ergospirometry of the heart failure patients: A) maximum cardiorespirat ory test time length (minutes), B) peak heart rate (bpm) and C) peak maximum oxygen consumption (peak $\left.\mathrm{VO}_{2}, \mathrm{ml}^{-\mathrm{kg}^{-1}} . \mathrm{min}^{-1}\right)$.

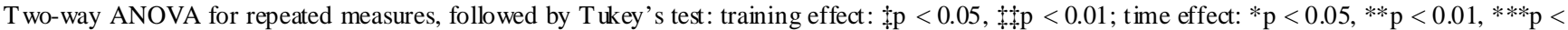
0.001

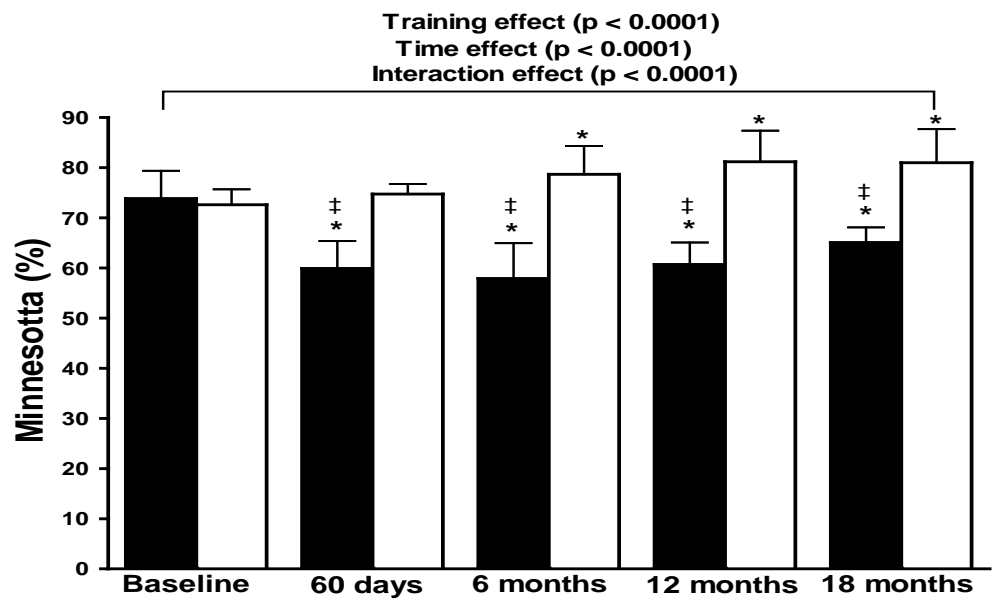

Figure 2. Supervised cardiac rehabilitation (full bars) and control (empty bars) effects on heart failure patients' quality of life by means of the "Minnesota Living with Heart Failure Questionnaire”. Two-way ANOVA for repeat ed measures followed by Tukey's test (mean \pm standard deviation): training effect: $\ddagger<0,05$; time effect: ${ }^{*}$ p $<0.05$

\section{Discussion}

The supervised CR program proposed in this study was effective and efficient, as seen by the high patient adherence in the proposed sessions (92\%), by the success in all of the proposed activities, cardiopulmonary improvement, symptoms reduction and quality of life improvement. On the other hand,control patients,which received standard care,justshowed improvement in the first 30 days in peak $\mathrm{VO}_{2}$ and maximum test time length, and at 60 days in physical capacity, probably because of the low adherence to standard care verified in this group (16\%).

\subsection{Supervised Program Effects}

In general, supervised CR programs are successful in increasing physical fitness, cardiovascular performance, quality of life and reducing mortality[5-13,19-21,2224].However, there are indications that only $11 \%$ to $30 \%$ of those who need $\mathrm{CR}$ have access to specialized centers $[6,22,23,25]$.Taking these facts into consideration, there has been an increase in the number of programs, whether supervised or not. The effectiveness of these programs has been well documented, revealing broad benefits[19,23,26-28],although other studies have failed to observe positive effects, especially those regarding quality of life[29].In the present study, patients with HF in both groups demonstrated improvement in cardiorespiratory fitness and in functional capacity during the 60 intervention days, as verified by other authors[19-21,23,2628].Nevertheless, only the supervised CR group showed quality of life improvement along the 60 CR days. As in other studies[30,31],quality of life benefits in CG patients were very discrete, likely because of low program adherence to standard care recommendations (16\%), negatively influencing the results in this group.

Some reasons may be highlighted for the benefits observed in CR program: motivation on behalf of the supervised program patients for attending a CR sector (department), specialized professional supervision, encouragement offered by the therapists during sessions, quality of service offered and social bonding with other patients in CR sessions. The differences verified when comparing both programs draw attention to the importance of the quality of the program offered, CR program adherence and adequate patient support[7,8,10,23,26,30,31].

\subsection{Cardiores piratory Effects}

Traditionally, maximum oxygen consumption $\left(\mathrm{VO}_{2} \max \right)$ 
is a determiner for individuals' physical and functional capacity. It is known that adequately planed intensity, time length and frequency of physical activities are the onlyeffective ways to improve $\mathrm{VO}_{2}$ max. Th is phenomenon is related to better cardiovascular and respiratory performance, higher muscular blood flow and greater number of mitochondria involved in aerobic metabolism[11,12,20]. Studies indicate a $10 \%$ to $50 \%$ increase in $\mathrm{VO}_{2} \max$ after $\mathrm{CR}$ program execution[5,6,11,21,30].Our results are similar to the ones cited - $18 \%$ peak $\mathrm{VO}_{2}$ max improvement in CR and $15 \%$ in control patients - indicating the effectiveness of our program.

\subsection{Quality of Life}

During the 60 days of the program, none of the patients had any cardiac episode, nor needed medication change. On the routine medical appointments, in general, patients referred better well-being sensation due to CR. These results are in consonance withthe benefits verified in cardiorespiratory and functional capacity, and on the quality of life evaluation categories (in CR), as foreseen by other authors[31].Throughout the 18 months follow-up period,quality of life category improvement was only verified in CR. It is very likely that cardiorespiratory and functional capacity imp rovements in consequence of regular physical activity practice produced better physical performance scores for daily life activities execution and subjective health perception. These results are consistent with other studies[6,7,10,26-28,32]and emphasize the importance of having exercise training programs for quality of life improvement in HF patients. Nevertheless, there are evidences that contradict these arguments. Wijkstra[33],for example, did not find significant correlation when comparing physiologic data and quality of life in patients submitted to rehabilitation, although they observed parallel improvement in those patients. In Pell's review[29],the author did not find significant rehabilitation program benefits on patients' quality of life. This matter is still under debate.

\subsection{Hos pital Admission and Mor tality}

Broad evidences endorse that $\mathrm{CR}$ is responsible for significant mortality reduction from all causes,from cardiovascular diseases, re-infarction and rehospitalization[1,2,5,6,7,10,21,29,31].These reductions are related to the functional and cardiorespiratory improvements, risk factors reduction and lifestyle improvement. In the present study, hospital admittance and death indexeswere lower in the supervised exercise training group, although these differences did not reach statistical significance. Merely the emergency service frequency use in CR group was significantly lower than CG at the end of 18 months follow-up. This perceptible contrast with the studies mentioned above may be caused by the small sample, as demonstrated by others[29]. Nonetheless, hospitalization reduction as well as emergency services can favorably affect costs in HF treatment, emphasizing CR treatment[2,5,6,7,10,23,24].

These findings bring about at least two new questions: $1^{\text {st }}$ - Which aspects are necessary to increase adherence to unsupervised CR programs? $2^{\text {nd }}$ - Which strategies should be adopted to extend the benefits found in short term programs?

\subsection{Limitations}

The present study has a few limitations. First, the fact of only having male subjects limits the conclusions drawn fro $m$ the study. Secondly, the reduced number of patients in each group and the fact that they were sent by a single medical service,also limits the extent of our conclusions.

\section{Conclusions}

The results of the present study allow us to conclude that a well-designedsupervised cardiac rehabilitation program is efficient and effective for heart failure patients. Card iorespiratory fitness, functional capacity and quality of life improvementshave occurred.Reduction in the need of emergency treatment and increase in survival in these patients were also observed, both duringthe treatment program, and also in the 18 months follow-up period. These data are related to patient adherence and to the intrinsic benefits of supervised cardiac program rehabilitation.However, further research is still necessary to investigate ways to increase adherence to unsupervised CR programs, as most patients do not have access to specialized centers.

\section{REFERENCES}

[1] S. A. Hunt, W. T. Abraham, M. H. Chin, A. M. Feldman, G. S. Francis, T. G. Ganiats, et al. ACC/AHA 2005 guideline update for the diagnosis and management of chronic heart failure in the adult: a report of the American College of Cardiology/American Heart Association Task Force on Practice Guidelines. Journal of American Collegeof Cardiology, vol.46, pp. e1-e82, 2005.

[2] T. Thom, N. Haase, W. Rosamond, V. J. Howard, J. Rumsfeld, T. Manolio, et al. Heart disease and stroke statistics - 2006 update: a report from the American Heart Association Statistics Committee and Stroke Statistics Subcommittee. Circulation, vol. 113, pp. e85-151, 2006.

[3] P. G. Agostoni, G. Cattadori, M. Bianchi, K. Wasserman. Exercise-Induced Pulmonary Edema in Heart Failure. Circulation, vol. 108. pp. 2666-71, 2003.

[4] D. V. Araujo, L. R.Tavares, R. Veríssimo, M. B. Ferraz, E. T. Mesquita. Cost of heart failure in the Unified Health System. ArquivosBrasileirosde Cardiologia, vol. 84. pp. 422-7, 2005.

[5] R. Belardinelli, D. Georgiou, G. Cianci, A. Purcaro. Randomized, Controlled Trial of Long-Term Moderate 
Exercise Training in Chronic Heart Failure: Effects on Functional Capacity, Quality of Life, and Clinical Outcome. Circulation, vol. 99, pp. 1173-82, 1999.

[6] P. A. Ades. Cardiac rehabilitation and secondary prevention of coronary heart disease. The New England Journal of Medicine, vol. 345, pp. 892-902, 2001.

[7] J. Austin, R. Williams, L. Ross, L. Moseley, S. Hutchison. Randomized controlled trial of cardiac rehabilitation in elderly. European Journalof Heart Failure, vol. 7, pp. 411-7, 2005.

[8] S. Jónsdóttir, K. K. Andersen, A. F. Sigurosson, S. B. Sigurosson. The effect of physical training in chronic heart failure. European Journal of Heart Failure, vol. 8, pp. 97-101, 2006.

[9] N. Marchionni, F. Fattirolli, S. Fumagalli, N. Oldridge, F. Del Lungo, L. Morosi, et al. Improved exercise toler ance and quality of life with cardiac rehabilitation of older patients after myocardial infarction: results of a randomized, controlled trial. Circulation, vol. 107, pp. 2201-6, 2003.

[10] J. Austin, W. R. Williams, L. Ross, S. Hutchison. Five-year follow-up findings form a randomized controlled trial of cardiac rehabilitation for heart failure. European Journal of Cardiovascular and Preventive Rehabilitation, vol. 15, pp. 162-7, 2008.

[11] A. A. Ehsani, G. W. Heath, J. M Hagberg, B. E. Sobel, J. O. Holloszy. Effects of 12 months of intense exercise training on ischemic ST-segment depression in patients with coronary artery disease. Circulation, vol. 64, pp. 1116-24, 1981.

[12] R. S. Taylor, A. Brown, S. Ebrahim, J. Jolliffe, H. Noorani, K. Rees, et al. Exercise-based rehabilitation for patients with coronary heart disease: systematic review and meta-analysis of randomized controlled trials. American Journal of Medicine, vol. 116, pp. 682-92, 2004.

[13] N. K. Wenger. Current Status of Cardiac Rehabilitation. Journal of American College of Cardiology, vol. 51, pp. 1619-31, 2008.

[14] World Medical Association. Declaration of Helsinki: Recommendation guiding physicians in biomedical research involving human subjects.Journal of AmericamMedical Association, vol. 277, pp. 925-926, 1997.

[15] K. L. Grady, K. Dracup, G. Kennedy, D. K. Moser, M. Piano, L. W. Stevenson, et al. Team management of patients with heart failure: A statement for healthcare professionals from The Cardiovascular Nursing Council of the American Heart Association. Circulation, vol. 102, pp.2443-56, 2000.

[16] A. G. Gift, G. Narsavage. Validity of the numeric rating scale as a measure of dyspnea. American Journal of Critical Care, vol. 7, pp. 200-4, 1998.

[17] K. Wasserman, J. E. Hansen, D. Y. Jue, B. J. Whipp. Principles of exercise testing and interpretation. Philadelphia: Lea and Febiger, 1987.

[18] T. S. Rector, S. H. Kubo, J. N. Cohn. Patients' selfassessment of their congestive heart failure: content, reliability, and validity of a new measure, the Minnesota Living with Heart Failure Questionnaire. Heart Failure, vol. 3, pp. 198-209, 1987.
[19] M. Godoy. National Cardiovascular Rehabilitation Consensus. Arquivos Brasileiros de Cardiologia, vol. 69, pp. 267-91, 1997.

[20] N. F. Gordon, M. Gulanick, F. Costa, G. Fletcher, B. A. Franklin, E. J. Roth, et al. Physical Activity, and Exercise Recommendations for Stroke Survivors: An American Heart Association Scientific Statement From the Council on Clinical Cardiology, Subcommittee on Exercise, Cardiac Rehabilitation, and Prevention; the Council on Cardiovascular Nursing; the Council on Nutrition, Physical Activity, and Metabolism; and the Stroke Council. Circulation, vol. 109, pp. 2031-41, 2004.

[21] A. S. Leon, B. A. Franklin, F. Costa, G. J. Balady, K. A. Berra, K. J. Stewart, et al. Cardiac Rehabilitation and Secondary Prevention of Coronary Heart Disease. An American Heart Association Scientific Statement From the Council on Clinical Cardiology (Subcommittee on Exercise, Cardiac Rehabilitation, and Prevention) and the Council on Nutrition, Phy sical Activity, and Metabolism (Subcommittee on Physical Activity), in Collaboration With the American Association of Cardiovascular and Pulmonary Rehabilitation. Circulation, vol. 111, pp. 369-76, 2005.

[22] J. A. Oliveira Filho, A. C. Leal, V. C. Lima, D. V. Santos Filho, B. Lun a Filho. Unsupervised Rehabilitation: effects of exercise training over long run. ArquivosBrasileirosde Cardiologia, vol. 79, pp. 239-44, 2002.

[23] J. J. Carlson, J. A. Johnson, B. A. Franklin, R. L. vanderLaan. Program Participation, Exercise Adherence, Cardiovascular Outcomes, and Program Cost of Traditional Versus Modified Cardiac Rehabilitation. American Journal of Cardiology, vol. 86, pp. 17-23, 2000.

[24] P. A. Ades, F. J. Pashkow, J. R.Nestor. Cost-effectiveness of cardiac rehabilitation after myocardial infarction. Journal or Cardiopulmonary Rehabilitation, vol. 17, pp. 222-31, 1997.

[25] I. A. Scott, K. A. Lindsay, H. E. Harden. Utilization of outpatient cardiac rehabilitation in Queensland. Medical Journal of Australia, vol. 179, pp. 341-5, 2003.

[26] H. M. Arthur, K. M. Smith, J. Kodis, R. McKelvie. A controlled trial of hospital versus home-based exercise in cardiac patients. Medicine and Science in Sports and Exercise, vol. 34, pp. 1544-50, 2002.

[27] H. M. Dalal, P. H. Evans, J. L. Campbell, R. S. Taylor, A. Watt, K. L. Read, et al. Home-based versus hospital-based rehabilitation after my ocardial infarction: A randomized trial with preference arms - Cornwall Heart Attack Rehabilitation Management Study (CHARMS). International Journal of Cardiology, vol. 119, pp. 202-11, 2007.

[28] R. S. Taylor, A. Watt, H. M. Dalal, P. H. Evans, J. L. Campbell, K. L. Read, et al. Home-based cardiac rehabilitation versus hospital-based rehabilitation: A cost effectiveness analysis. International Journalof Cardiology, vol. 119, pp. 196-201, 2007.

[29] J. Pell. Cardiac Rehabilitation. Coronary Health Care, vol. 1, pp. 8-17, 1997.

[30] D. R. Thompson. Cardiac Rehabilitation: how can it be improved? Journal of Psychosomatic Research, vol. 39, pp. 519-23, 1995.

[31] D. R. Thompson, G. S. Bowman. Evidence for the 
Effectiveness of Cardiac Rehabilitation. Clinical Effectivenessin Nursing, vol. 1, pp. 64-75, 1997.

[32] B. A. F. van Tol, R. J. Huijsmans, D. W. Kroon, M. Schothorst, G. Kwakkel. Effects of exercise training on cardiac performance, exercise capacity and quality of life in patients with heart failure: A meta-analysis. European
Journal of Heart Failure, vol. 8, pp. 841-50, 2006.

[33] P. J. Wijkstra, R. Van Altena, J. Kraan, V. Otten, D. S. Postma, G. H. Koeter. Quality of life in patients with chronic obstructive pulmonary disease improves after rehabilitation at home. European Respiratory Journal, vol. 7, pp. 269-73, 1994. 